\title{
LAS SACRAS HISPANAS PRETRIDENTINAS: HISTORIA DE UN PRODUCTO EDITORIAL HUIDIZO
}

\author{
POR \\ Helena Carvajal GonzÁlez ${ }^{1}$ \\ Universidad Complutense de Madrid
}

\begin{abstract}
RESUMEN
Las sacras fueron objetos habituales sobre la mesa de altar desde la segunda mitad del siglo XVI y hasta el Concilio Vaticano II. Este hecho ha provocado que se consideraran emanadas de las disposiciones tridentinas, aunque el origen de estas tablillas con textos litúrgicos es bajomedieval. El artículo analiza su forma, usos y evolución entre la Baja Edad Media y la primera Edad Moderna, así como las razones de su pobre conservación.
\end{abstract}

PALABRAS CLAVE: sacras; tabella secretarum; manuscritos litúrgicos; impresos litúrgicos.

\section{HISPANIC PRETRIDENTINE ALTAR CARDS: HISTORY OF AN ELUSIVE EDITORIAL PRODUCT}

\begin{abstract}
Tabellae secretarum were usual objects on the altar table from the second half of the 16th century until the Second Vatican Council. This fact caused them to be considered as emanating from the Tridentine dispositions, although the origin of these tablets with liturgical texts is late medieval. The article analyses their form, uses and evolution between the Late Middle Ages and the Early Modern period, as well as the reasons for their poor conservation.
\end{abstract}

KEY WORDS: altar cards; tabella secretarum; liturgical manuscripts; liturgical prints.

Cómo CITAR eSTe ARTículo / CitATION: Carvajal González, Helena. 2020. "Las sacras hispanas pretridentinas: historia de un producto editorial huidizo». Hispania Sacra LXXII, 146: 451-459. https://doi.org/10.3989/hs.2020.032

$\begin{array}{ll}\text { Recibido/Received } & 16-04-2019 \\ \text { Aceptado/Accepted } & 26-11-2019\end{array}$

Las sacras, llamadas también Tabellae secretarum ${ }^{2}$ o Charta cum secretis, ${ }^{3}$ son tablillas con textos de la misa que se colocaban sobre la mesa de altar para ayudar al sacerdote a recitar las oraciones principales del oficio, sin tener que recurrir al misal en momentos en los que debía inclinarse o alzar las manos. ${ }^{4}$ Su contenido y uso ha variado sensiblemente a lo largo de la historia, adaptándose a los

\footnotetext{
1 hcarvajal@ucm.es / ORCID iD: https://orcid.org/0000-0001-9683-4234

2 Cartaglorias en italiano, altar cards en inglés, kanontafel en alemán. En México se denominan «palabreros»; véase Pomar 2012, 484, nota 24.

3 Martí 2013, 200.

4 Righetti 1955, II: 498.
}

sucesivos cambios litúrgicos y, aunque en la actualidad apenas se emplean, ${ }^{5}$ con anterioridad al Concilio Vaticano II las rúbricas del misal romano ordenaban la colocación de una tablilla en el centro del altar, a los pies de la cruz: "Ad Crucis pedem ponatur Tabella Secretarum appellata». ${ }^{6}$ Esta tabla central generalmente contenía textos del Canon de la misa, el ofertorio y la consagración —oración que el sacerdote recitaba en voz baja, de ahí la denominación de este objeto-, el Gloria y el Credo, entre otros; además,

\footnotetext{
5 La editorial COCULSA imprimió todavía en 1969 ejemplares de sacras en castellano.

6 Missale Romanum, ex decreto Sacrosancti Concilii Tridentini restitutum..., Ex Officina Plantiniana, etc., 1627, h. c8v.
} 
fue frecuente desde el siglo XVII colocar a la derecha del celebrante, en el lado de la Epístola, la oración del Lavabo (Lavabo inter innocentes manus meas...) y, a la izquierda, los primeros versículos del Evangelio de Juan. ${ }^{7}$ Durante la exposición del Santísimo Sacramento, debían ser retiradas del altar. $^{8}$

Aunque su auge se ha vinculado repetidas veces a la reforma tridentina, ${ }^{9}$ se conservan ejemplos impresos del siglo XV en Alemania ${ }^{10}$ y en los inventarios de instituciones religiosas se da noticia de la existencia de tablillas o folios manuscritos, denominados a veces Te igitur, que se colocaban sobre el altar ya desde el siglo XIII, ${ }^{11}$ como más adelante se verá. Algunos autores, incluso, relacionan su incierto origen con los dípticos consulares tardoantiguos. ${ }^{12}$

Las fuentes documentales indican que, ya desde la primera mitad del siglo XVI, las sacras fueron una tipología de impreso de amplia difusión y uso. Muestra de la enorme importancia y demanda que las instituciones religiosas hicieron de este producto editorial se encuentra en el inventario de los bienes de Jacobo Cromberger, realizado en 1528 , en el que se contabilizan «37 manos de teygitur», lo que, a 25 pliegos por mano, arroja un total de 925 sacras. ${ }^{13}$ También en la relación de la venta de la imprenta de Jorge Coci a Bartolomé de Nájera y Pablo Hurus en 1536 se señala la existencia de un gran número de estos impresos, en concreto «cient sacras de pargamino, a razón de ocho dineros la pieça. E mas quatrozientas veintizinco sacras de papel a razón de dos dineros». ${ }^{14}$ Unos años más tarde, en 1544, el cabildo sevillano adquirió al impresor Juan Varela de Salamanca para el servicio de las diversas capellanías 200 tabellae en papel a 8 maravedís y 26 en pergamino a $51 .^{15}$

Estas referencias por sí solas ya confirman que el uso de las sacras no está ligado a las disposiciones tridentinas sino que se trata de un hábito anterior que, además, hunde sus raíces en la tradición manuscrita medieval. Como señala José de Villamil, estos primeros ejemplos se conocían con el nombre de Te igitur o teigitur, ${ }^{16}$ en referencia a las palabras iniciales del Canon missae "Te igitur clementissime Pater» que, como ya se ha mencionado al inicio, se incluían con frecuencia en la tabla central. Uno de los te igitur más antiguos localizados es el que formaba parte de los bienes inventariados en la catedral de Salamanca en $1275 .{ }^{17}$ Ángel Riesco, al referirse a este ejemplar, señala que:

Concluida la plegaria del prefacio y tras la recitación del "sanctus» de la misa, el celebrante, con los brazos extendidos hacia arriba, fijaba los ojos en el crucifijo

\footnotetext{
7 Giorgi 2008, 29. Brouns 2005, 480.

8 Sacra Congregazione dei Riti, decr. 3130 ad 3. Véase Oppenheim 1949, coll. 956-957.

9 Brancato y Pontillo 2016, s.p. Oppenheim 1949, coll. 956-957; Schulte 1913.

10 Schmidt 2010.

11 Villamil 1906, 74; Jungmann 1962, 1: 77, nota 1.

12 Brancato y Pontillo 2016, s.p

13 Griffin 1988, 214.

14 Pedraza 2016, 208-209. El documento fue dado a conocer por Abizanda 1915-1930, I: 311.

15 Álvarez Márquez 1992a, 136 y 146.

16 Villamil 1906, 74.

17 Ídem, reproduciendo el originalmente publicado por GarcíaMoreno 1902, VII: 175-179. También ha sido estudiado más recientemente por Riesco 1996.
}

para iniciar, con las palabras "Te igitur», la recitación del canon. El texto completo de esta plegaria solía colocarse en lugar bien visible sobre una tablilla, a modo de "sacra», situada directamente sobre el altar o colgada de alguna repisa en el retablo. ${ }^{18}$

La pintura del siglo $\mathrm{XV}$, extremadamente minuciosa en la representación de los espacios interiores, suele plasmarlas con frecuencia en escenas de celebración litúrgica, como las de la Misa de San Gregorio. A veces aparecen como una simple hoja con un breve texto que se coloca a los pies de la cruz (Fig. 1), otras en forma díptico (Fig. 2) o tríptico (Fig. $3)$. A veces, incluso, se las ve protegidas por un discreto marco, como se aprecia en el Libro de horas de Enrique VIII de Francia (Fig. 4).

Ya a finales de la Edad Media las menciones a las sacras son abundantísimas. En el inventario realizado en 1494 en la iglesia parroquial de Santa Ana de Guadalcanal (Sevilla) se menciona que en el altar mayor, debajo de la imagen de la Santa Ana triple que preside el templo, se encuentra "un Te igitur grande, viejo, roto». ${ }^{19} \mathrm{~A}$ inicios del XVI se puede citar el de la parroquia de Santiago de Calahorra, situado en el altar de San Llorent junto a un «retablo común». ${ }^{20}$ La propia reina Católica envió a su hija doña María diecisiete libros y un pergamino con las palabras de la Consagración ${ }^{21}$. Años más tarde, en el inventario de otra de sus hijas, doña Juana I, de 1545 se menciona, junto a velas, misales y otros objetos litúrgicos «un teygitur que se hizo para la capilla de su alteza». ${ }^{22}$

Algunos documentos aluden al soporte en el que se escriben o imprimen estos objetos, como el «te igitur de pergamino" que aparece en el inventario de Santa Lucía de Alcuescar, realizado en $1500,{ }^{23}$ el «te igitur de pargamino mui bueno enquadernado en tablas de cuero colorado el qual dio don Favian Justiniano thesorero desta iglesia (Santa María la Mayor de Valladolid)» ${ }^{24}$ en 1547, o el «teigitur de papelón» que poseía Antonio Romero, chantre de Traspinedo a su muerte en $1577 .{ }^{25}$ A veces, incluso, conviven diversas tipologías como sucedía en el monasterio cántabro de Santa María de Piasca, donde, en 1519, el visitador fray Gaspar de Villaroel, abad de Sahagún, inventarió «un te igitur de molde y otro de pergamino y pintado». ${ }^{26}$ Las fuentes parecen indicar que estas tablas con diversos textos litúrgicos, en estos años aún no del todo codificados como sucederá después de Trento, convivían con otras de tipo organizativo que regían la vida interna de los templos. Álvarez Márquez señala que a principios del XVI todas las capillas de la Catedral de Sevilla contaban, al menos, con un misal y «unas tablas del 'Te

\footnotetext{
18 Ibíd., 296.

19 Serrano 1915, 45.

20 Lecuona 1952, 480.

21 Ruiz 2004, 230.

22 Ferrandis $1943,3,234$

23 Martín 2014, 1536.

24 Rojo Vega, Anastasio. 1547. El sacristán Sancho de Grijalba y los libros de la colegiata de Santa María la Mayor de Valladolid. Patrimonio Nacional: Investigadores. En línea <https://investigadoresrb.patrimonionacional.es/uploads/2013/06/1547-SANTA-MARIA. pdf $>$ (Consulta 27-10-2018).

25 Rojo Vega, Anastasio. Testamento, inventario y biblioteca de Antonio Romero, chantre (1577). Patrimonio Nacional: Investigadores. En línea <https://investigadoresrb.patrimonionacional. es/node /5815 > (Consulta 27-10-2018).

26 Solórzano 2007, 347.
} 
FIGURA 1

Misa de San Gregorio, S. XV, Fine Arts Museum of San Francisco
FIGURA 2

Misa de San Gregorio, Juan de Nalda, S. XV, Museo Arqueológico Nacional
FIGURA 3

Misa de San Gregorio, Pedro Berruguete, 1490, Museo de Burgos
FIGURA 4

Misa de San Gregorio, Jean Poyer, Libro de horas de Enrique VIII de Francia (c. 1500), Morgan Library \& Museum, MsH.8, f. 168r
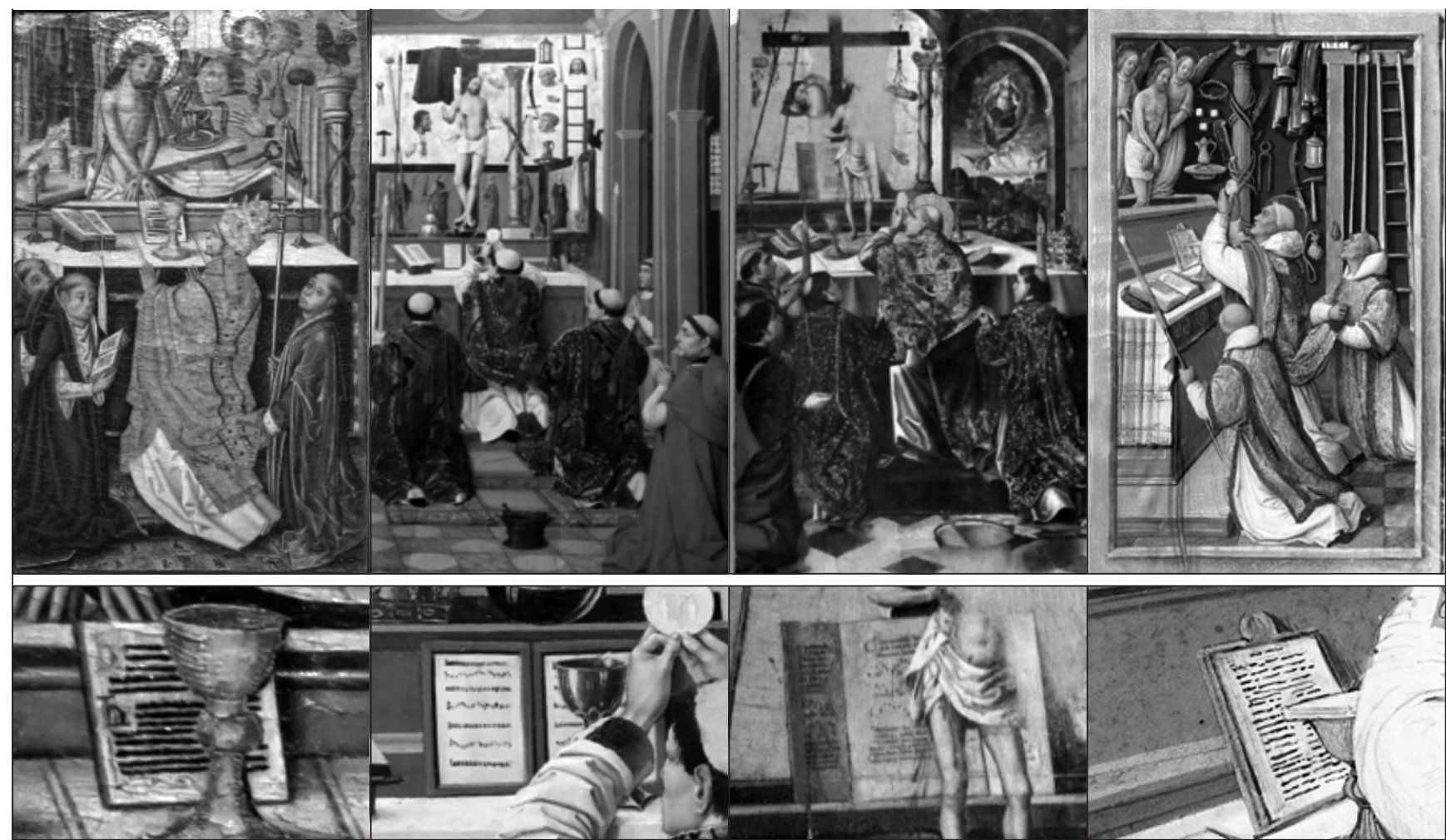

Fuentes: Figuras 1 y 3: Wikimedia Commons. Figura 2: autora. Figura 4: Morgan Library \& Museum, licencia CC BY-NC-SA 4.0.

igitur'» pero, además de estas, existían otros textos colocados sobre un soporte rígido en el que se establecían, por ejemplo, las capellanías de la Iglesia, los altares donde se había de decir misa y los capellanes que debían cantarla, las oraciones con las que se visten los sacerdotes o el orden con el que se debía guardar el silencio en las capillas. ${ }^{27}$ No parecen haber sobrevivido demasiados ejemplos medievales y modernos de este otro tipo de tablillas, aunque destacan algunos como el conocido «bojarte» o tabla de misas de la Cartuja de Miraflores en Burgos, de mediados del siglo XV y superviviente del incendio que asoló el monasterio en 1452. Ya en plena Edad Moderna se registra el uso de las tablas eclesiásticas, espacios que las autoridades religiosas emplearon para hacer pública determinada información de interés (fiestas de guardar, excomulgados, etc.). ${ }^{28}$

En muchas ocasiones, estas piezas aparecen listadas entre los libros litúrgicos lo que ha provocado identificaciones erróneas por desconocimiento de su función. Es el caso del "teigitur e prefacios pintados» y el «teigitur de cantoría nuevo" que se mencionan entre los bienes de las encomiendas de la Orden Santiaguista en 1507 y que han sido considerados libros por los autores que los han estudiado. ${ }^{29}$

Finalmente, existen menciones a su uso en la liturgia. En el reciente estudio de Enrique Llopis y Elisa Ruiz, El

\footnotetext{
27 Álvarez 1992b, 24-26.

28 Castillo 2006, 214-218 y 2009, 583.

29 Ortiz 1995, 62 y 64.
}

monasterio de Guadalupe y la Inquisición, se menciona que precisamente la forma poco reverente de recitar lo contenido en las sacras fue empleado como prueba acusatoria contra contra fray Diego de Burgos el Viejo:

[Testificación de fray de fray Luis de Córdoba contra
fray Diego de Burgos el Viejo]
Otra vez, en este mesmo altar [de santa Ana], desque
avía dicho el Evangelio, començó a dezir luego las sacras. E
respondiéndole al prephaçio, desque yo vi que proseguía
adelante, díxele: «¿Non fazéis la ofrenda?» Respondiome
entonçes rezio que qué ofrenda. E volvió la cara e vio el
cáliz como lo avía dexado fecho. E feriose en los pechos. ${ }^{30}$

Aunque no se conocen hasta la fecha ejemplos hispanos medievales iluminados, los datos provenientes de los inventarios y los paralelos encontrados en otros países europeos hacen suponer que aquellas sacras realizadas para las sedes más prestigiosas estarían ilustradas, como lo están los ejemplos manuscritos sobre pergamino del siglo XVI custodiados en diversos centros - es el caso de la sacra conservada la British Library, iluminada para un convento de monjas del norte de Italia, ${ }^{31}$ o la vendida por Maggs Bros. Ltd. que

30 Llopis y Ruiz 2019, 541. Agradezco a la Dra. Ruiz la noticia de este dato de su reciente trabajo.

31 Esta sacra del siglo XVI, iluminada con la imagen de un obispo nombrando a una abadesa, constituye el fol. 128 de un álbum misceláneo con signatura BL Additional 41996. Puede consultarse en línea en <https://www.bl.uk/catalogues/illuminatedmanuscripts/record.asp? MSID=18981\&CollID=27\& NStart=41996 > (Consulta: agosto de 2019). 
FigurA 5a

Tabella secretarum manuscrita, S. XVI, vendida por Maggs Bros. Ltd, Londres
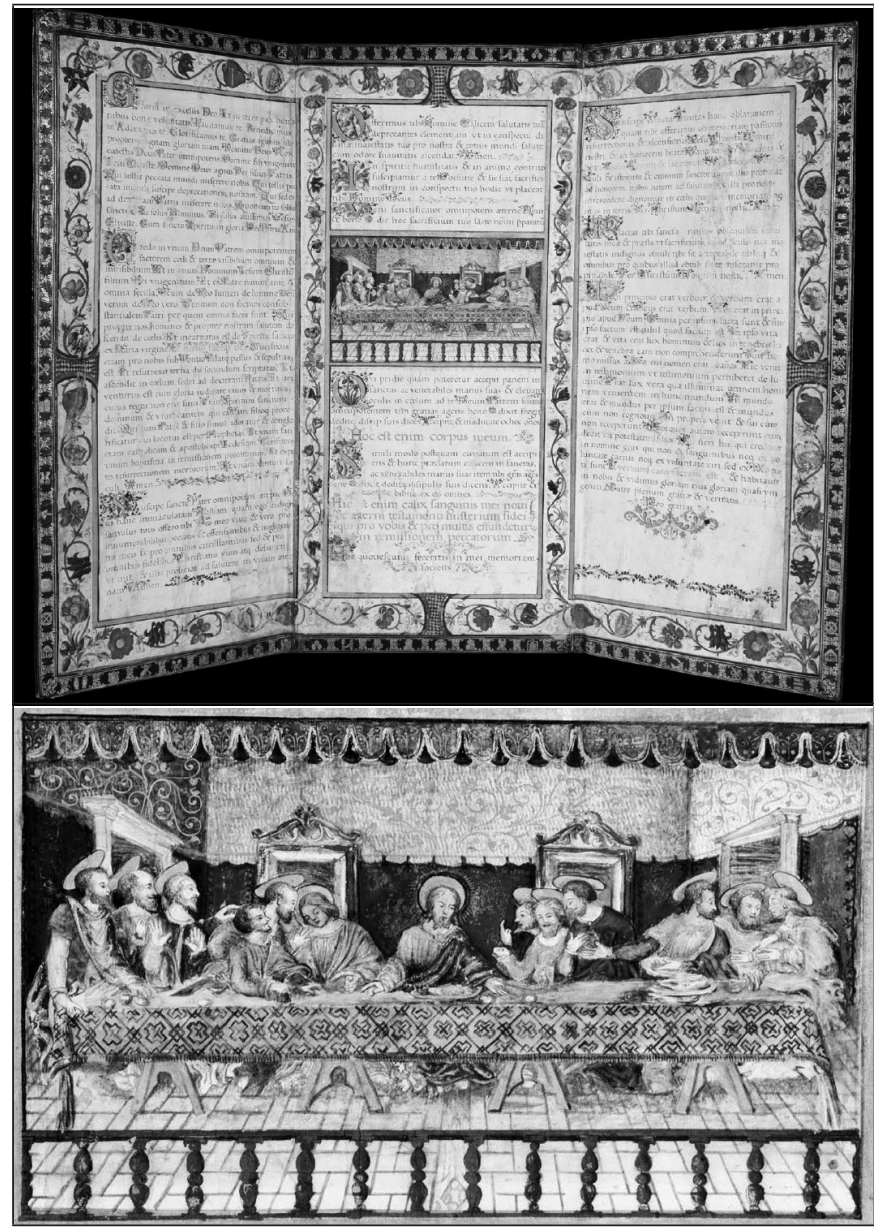

Fuente: https://www.maggs.com

reproduce la Santa Cena de Leonardo Da Vinci- ${ }^{32}$ y luego lo estarán los impresos, frecuentemente con una xilografía del Calvario siguiendo la tradición de la miniatura de la crucifixión que, desde la Alta Edad Media, acompañaba el inicio del Te igitur en los misales (Fig. 5).

En este sentido, Ma Carmen Álvarez Márquez señala que la Catedral de Sevilla poseía en el siglo XVI cuatro ejemplares ricamente iluminados y guarnecidos. En concreto:

La cuarta sacra con el «Te igitur» estaba también iluminada y guarnecida por dentro y por fuera, e incluso por los cantos, con cenefas y molduras de plata, doradas y estampadas, con cuadros esmaltados de diferentes colores en campo de plata a lo largo del friso, todo ello se hallaba claveteado sobre la madera con clavos y tachuelas de plata. Las tablas trababan entre sí mediante cantoneras, bisagras y botones de hierro dorados y las puertas tenían un botón de plata dorado cada una para abrirlas. ${ }^{33}$

Sin embargo, la mayor parte de los ejemplares fabricados y comercializados no serían piezas lujosas como las que se hallaban en la catedral hispalense, sino que estarían al

32 MAGGS BROS. LTD. RARE BOOKS AND MANUSCRIPTS, Eight remarkable works of illumination from 1470 to 1700, [Londres, Maggs Bros., c. 2010], (Illuminated books, manuscripts and miniatures), p. [6-7]

33 Álvarez 1992a, 113-114.
FIGURA 5b

Tabella secretarum impresa, S. XVI, BNE R/41699

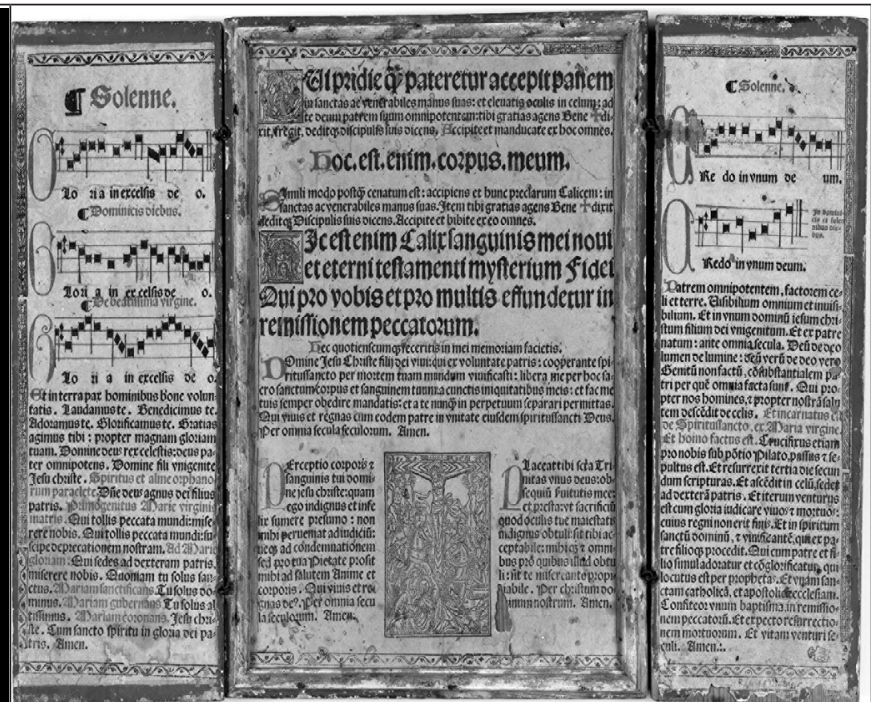

Fuente: BNE. Licencia CC BY-NC-SA 4.0

alcance de todas las economías, por modesta que fuera la parroquia. Como indican los documentos antes citados, la comercialización de las sacras se haría a precios modestos: 8 dineros para una sacra en pergamino y dos para una de papel del taller zaragozano de Jorge Coci; ${ }^{34}$ es decir, la mitad de lo que costaba una cuerna de pan fabricada en el horno canonical durante el s. XVI en Barcelona. ${ }^{35}$

Un interesante documento de la década de los 70 del siglo XVI, muestra cómo Gabriel de Hijar, administrador de los libros del Nuevo Rezado en Aragón, introduce en este territorio un total de 1.000 sacras, probablemente destinadas a actualizar las pretridentinas que hubiera, al tiempo que se envían a Castilla 500 producidas en Aragón. ${ }^{36}$ Estos movimientos de sacras después del Concilio de Trento sugieren que la sustitución por ejemplares más modernos y actualizados sería una de las causas de la pobre preservación de los ejemplares incunables y postincunables.

Se conservan actualmente en la Península unos pocos ejemplos anteriores al Concilio de Trento: una sacra manuscrita sobre pergamino actualmente custodiada en el Museo de Villafranca del Cid (Castellón) (Fig. 6); un fragmento

34 Pedraza 2016, 208-209. El documento fue dado a conocer por Abizanda 1915, 311.

35 Feliu 1991, I: 31.

36 Pedraza 1991, doc. 25. 
FIGURA 7

Fragmento de sacra impresa sobre pergamino, Zaragoza, Jorge Coci, AHN, Inquisición, MPD. 442

Sacra manuscrita sobre pergamino S. XV, Villafanca del Cid, Castellón

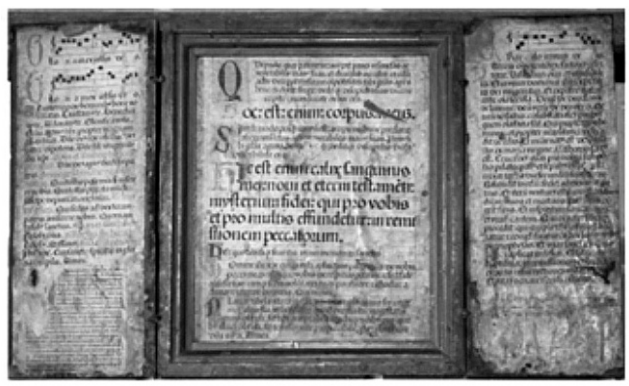

Fuente: fotografía autora.

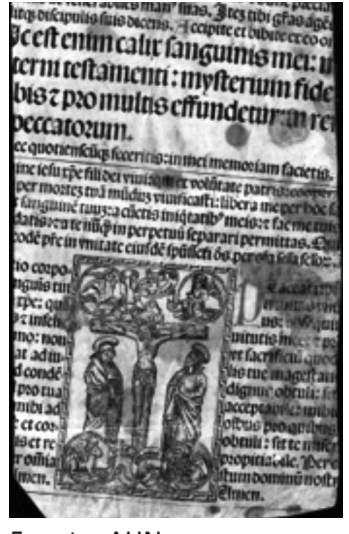

Fuente: AHN.
FIGURA 8

Sacra impresa sobre papel, BNE, $R / 41699$

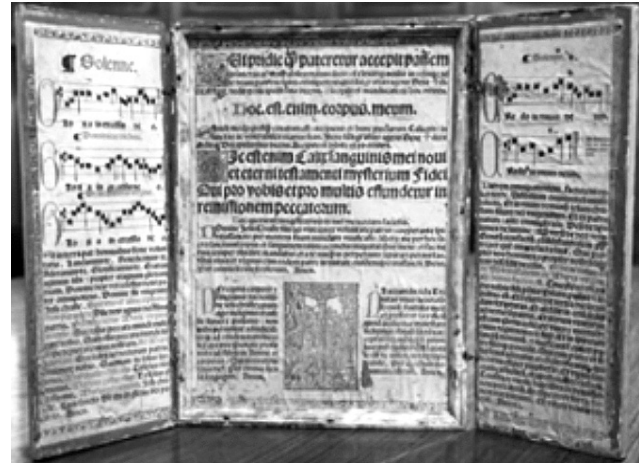

Fuente: BNE. Licencia CC BY-NC-SA 4.0. de principios del XVI conservado en el Archivo Histórico Nacional y recientemente identificado ${ }^{37}$ (Fig. 7), y la sacra impresa con ilustración xilográfica de la crucifixión, iniciales ornamentadas y notación musical cuadrada que adquirió la Biblioteca Nacional de España en $2016^{38}$ (Fig. 8).

La conservada en Villafranca del $\mathrm{Cid}^{39}$ tiene forma de tríptico y aunque la bibliografía generalmente la ha clasificado como impresa sobre pergamino ${ }^{40}$ o papel, ${ }^{41}$ el análisis in situ y las diferencias existentes entre letras que deberían ser idénticas indican que se trata, en realidad, de un texto manuscrito en letra gótica libraria de gran perfección, a dos tintas y con iniciales ornamentadas, probablemente del último tercio del siglo XV (Fig. 9). Este ejemplar acaso sea el más antiguo conocido hasta la fecha en España de este tipo de objetos litúrgicos, si bien no se puede descartar que otros puedan ir apareciendo, reutilizados de diversas formas. De hecho, según indica Rafael Monferrer la pieza

37 Carvajal 2019.

38 En línea: <http://www.bne.es/es/Inicio/Perfiles/Bibliotecarios/ NoticiasBibliotecarias/1020-Biblioteca-Nacional-adquiere-tablatriptico-siglo-XVI.html> (Consulta: octubre de 2019).

${ }_{39}$ Quiero agradecer a D. Guillem Monferrer Milian, Técnico de Turismo del Ayuntamiento de Villafranca, su amabilidad y las facilidades ofrecidas para realizar la consulta de la sacra castellonense.

40 En línea: <http://www.bne.es/es/Inicio/Perfiles/Bibliotecarios/ NoticiasBibliotecarias/1020-Biblioteca-Nacional-adquiere-tablatriptico-siglo-XVI.html> (Consulta: octubre de 2018); Monferrer 1986, 90; Milián 2013, 344: «Tríptico sacra. Iglesia parroquial. Es de madera y hoja de pergamino pegado; tipos de letra góticos del siglo XV; mide la central $32^{\prime} 5 \times 28 \mathrm{~cm}$ y las otras dos lo mismo que ésta ambas unidas. La primera comprende el Gloria, con iniciales rojas y notas de entonación del mismo, el resto del texto en negro; la segunda o del centro contiene la Consagración, iniciales a dos tintas en rojo y verde (!), y usando tipo mayor para las palabras de la consagración, y la tercera contiene el credo con iniciales y entonación en tinta roja y el resto en negro. Es uno de los ejemplares raros que se conocen, a pesar de ser utensilio tan sencillo. Mérito arqueológico extraordinario».

41 Valencia. Conselleria de Educación, Investigación, Cultura y Deporte «Resolución de 4 de noviembre de 2016, de la Consellería de Educación, Investigación, Cultura y Deporte, por la que se incoa expediente para declarar bien de interés cultural, con la categoría de monumento, la iglesia parroquial de Santa María Magdalena, sita en Vilafranca (Castellón)», Diario Oficial de la Generalitat Valenciana, num. 7925, 25-11-2016, p. 32957 fue recuperada de un desván, a donde probablemente fue a parar cuando quedó obsoleta, sustituida por alguno de los abundantes juegos de sacras más modernas que aún se conservan hoy en la parroquia de Villafranca del Cid. ${ }^{42}$

FIGURA 9

Sacra manuscrita abierta S. XV

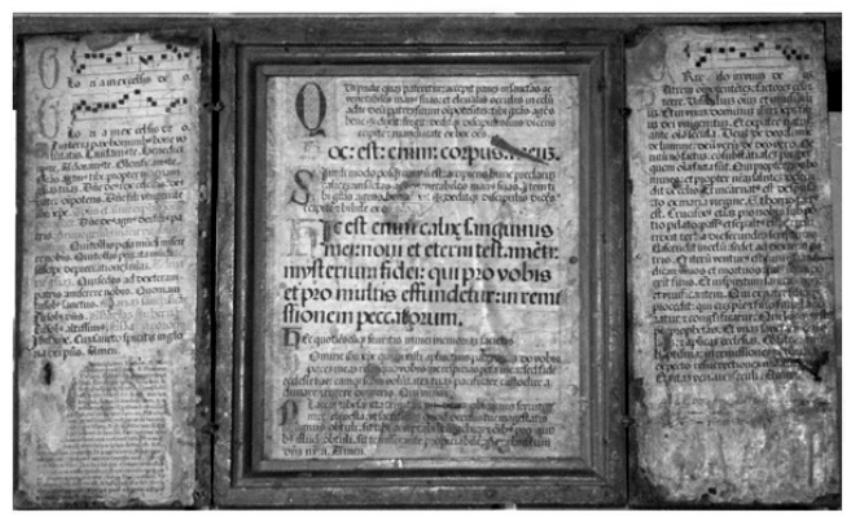

Fuente: Museo Parroquial de Villafranca del Cid (Castellón). Fotografía autora.

Se compone de una tabla central de $330 \times 280 \mathrm{~mm}$ en la que se enmarca, mediante una discreta moldura, un fragmento de pergamino de $258 \times 210 \mathrm{~mm}$. Esta tablilla central contiene el texto de la consagración desde «Qui pridie quam pateretur» hasta "sit te miserante propiciabile. Per Christum Dominum Nostrum. Amen». Sobre la central, con pequeñas bisagras, montan dos tablas laterales de aprox. $330 \times 140$ $\mathrm{mm}$ que cierran con una escarpia. La tabla izquierda contiene el texto del Gloria y la derecha el Credo, con notación cuadrada gregoriana sobre tetragrama rojo (Fig. 10).

En la parte inferior interna de la tablilla izquierda se ha pegado un fragmento de papel impreso en letra redonda; quedan también restos en la parte exterior de esta misma tabla de otro fragmento de impreso. Este hecho resulta interesante pues pone de manifiesto la actualización que sufrió la sacra, probablemente después de Trento, para

\footnotetext{
42 Monferrer 1986, 90.
} 
FIGURA 10

Sacra manuscrita sobre pergamino, S. XV. Detalle de la tabla derecha

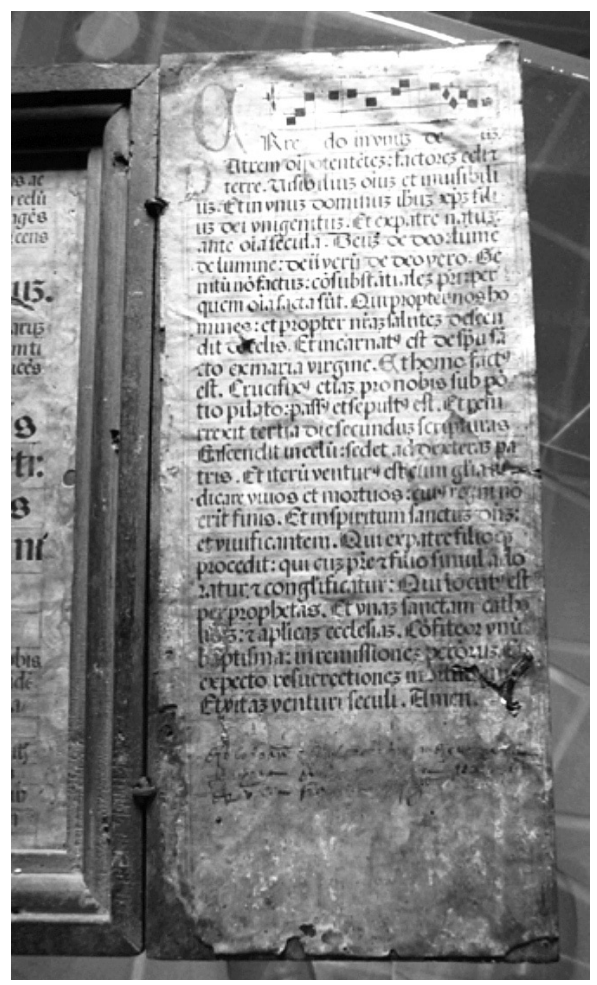

Fuente: Villafranca del Cid, Castellón. Fotografía autora.

poder seguir siendo empleada conforme a los usos litúrgicos del momento. En concreto, en la parte interior de la tabla izquierda se ha añadido el comienzo del Evangelio de Juan, desde «In principio erat verbum» hasta "plenum grátiæ et veritatis», texto que, como se ha señalado al inicio, fue habitual en las sacras desde principios del XVII (Fig. 11). Por fuera se ha adherido un fragmento del Salmo LXVI.

\section{FIGURA 11}

Sacra manuscrita sobre pergamino, S. XV. Detalle del impreso adherido a la tabla izquierda de la sacra con el comienzo del Evangelio de Juan

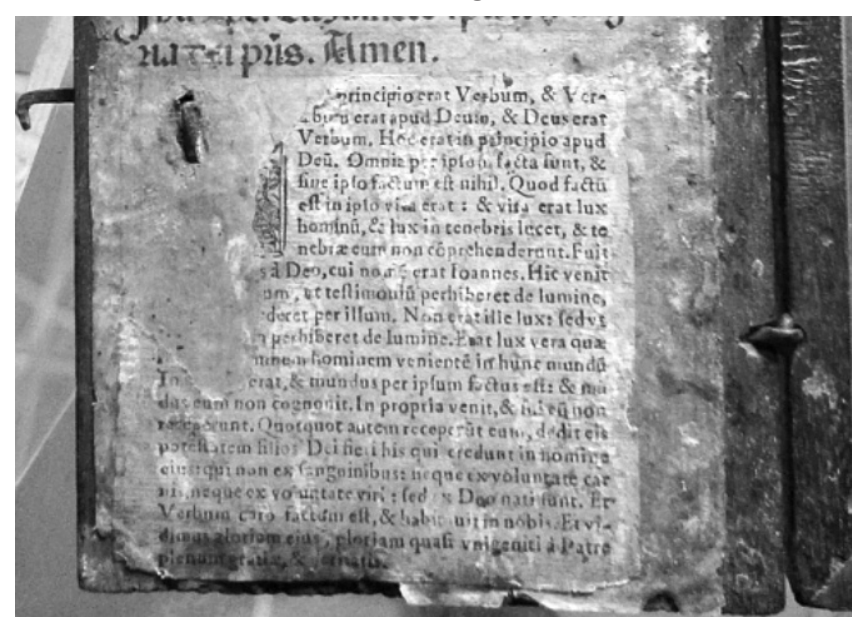

Fuente: Villafranca del Cid, Castellón. Fotografía autora.
El ejemplar de la Biblioteca Nacional de España ${ }^{43}$ fue adquirido por la Biblioteca a la librería zaragozana «Luces de Bohemia» en 2016, procedente de una colección particular $^{44}$ (Fig. 12).

\section{FIGURA 12}

Sacra impresa sobre papel S. XVI

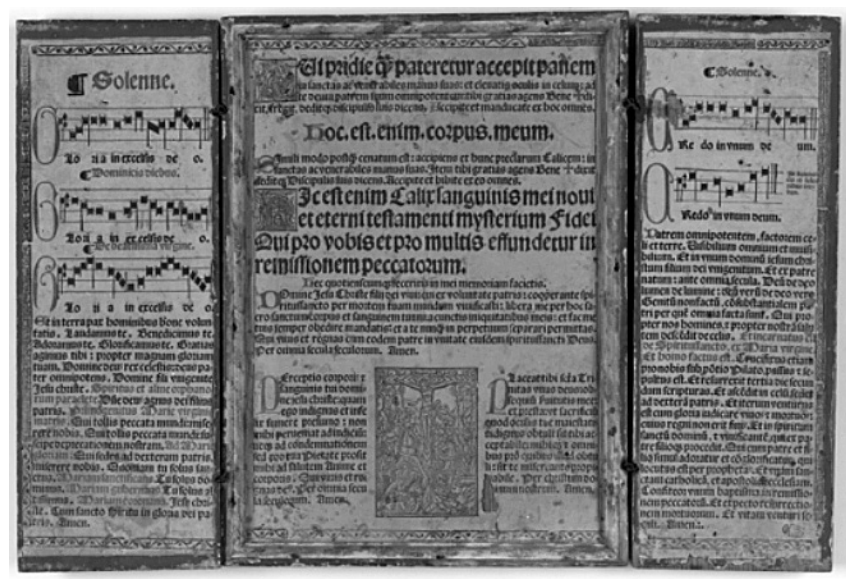

Fuente: BNE, R/41699. (Licencia CC BY-NC-SA 4.0)

Está formado también por tres tablas de madera, pintadas de negro en el exterior y rojo en el interior, con marmolado amarillo en la moldura que enmarca la tabla central. Dicha lámina central mide 235 × 310 mm y las laterales que montan sobre ella 117 × $310 \mathrm{~mm}$ cada una. Sobre la madera se ha adherido un papel impreso en tipografía gótica a dos tintas. Se han empleado también iniciales xilográficas sencillas en rojo y dos capitales xilográficas figurativas cuadradas, de $25 \mathrm{~mm}$ de lado cada una, con sendas imágenes de San Pablo, identificable por la espada y el libro, y la Vera faz, ambas con un perceptible desgaste por el uso.

En la parte inferior de la tabla central aparece un grabado de $60 \times 85 \mathrm{~mm}$ enmarcado por un sencillo filete con una detallada crucifixión en la que se identifican, además del Crucificado, a los dos ladrones, las santas mujeres acompañando a María desvanecida y San Juan evangelista.

Flanquean la parte central de la composición varios militares romanos entre los que destaca Longinos a la izquierda. El fondo, con un paisaje urbano, y, en general, toda la composición recuerda notablemente a modelos centroeuropeos contemporáneos (Fig. 13). Como ya se ha indicado, el uso de la xilografía de la crucifixión parece continuar una práctica habitual de los iluminadores que desde la Alta Edad Media aprovechaban la T inicial de la oración eucarística para incluir la imagen de la cruz en los misales.

43 Deseo agradecer al servicio de Reserva Impresa, en especial a D. Pilar Egoscozábal y al personal de la Sala Cervantes de la BNE, las facilidades ofrecidas para la consulta del ejemplar.

44 Anuncio de formalización de contrato. Número de Expediente 2016C0024NS3. Publicado en la Plataforma de Contratación del Estado el 12-07-2016 https://contrataciondelestado.es/wps/wcm/connect/ ace05a19-f21b-491d-b579-3bded11ea908/DOC_FORM2016-275060. pdf?MOD=AJPERES 
FIGURA 13

Tabella secretarum impresa, siglo XVI. Detalle de la xilografía de la crucifixión

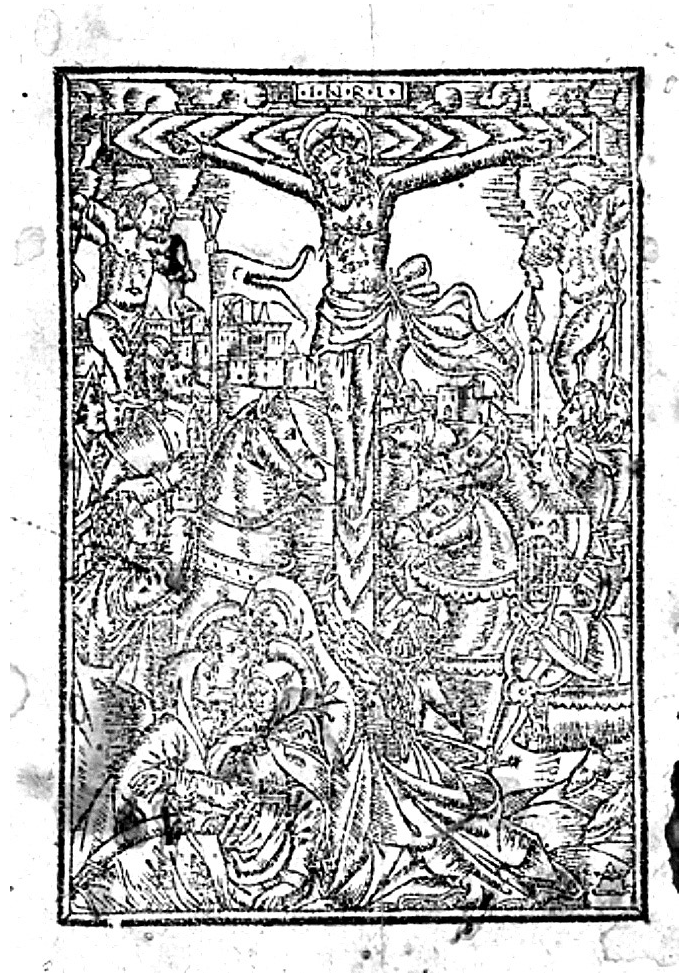

Fuente: Biblioteca Nacional de España. (Licencia CC BY-NC-SA 4.0)

El texto de cada tablilla lateral aparece enmarcado en el borde externo por diferentes fragmentos de orlas xilográficas vegetales y con decoración a candelieri yuxtapuestas de forma aleatoria. La central se enmarca arriba y abajo por estos mismos motivos y en los laterales por un sencillo filete.

En la tabla izquierda aparece el texto del Gloria en 28 líneas, con notación cuadrada, aparentemente xilográfica, en el que se han incluido diversos tropos como el Spiritus et alme orphanorum paraclete correspondientes a la misa De Beatissima virgine. La tabla central contiene las palabras de la consagración desde Qui pridie quam pateretur accepit panem in sanctas ac venerabiles manus suas... hasta mei memoriam facietis. Sigue la Oratio ante communio: Domine lesu Christe, Fili Dei vivi... hasta ...in unitate eiusdem Spiritus Sancti Deus. Per omnia saecula saeculorum Amen. A la izquierda del grabado continúa: Perceptio corporis et sanguinis tuis... hasta Per omnia secula seculorum. Amen. A la derecha del grabado, la oración del final de la misa: Placeat tibi, Sancta Trinitas... hasta ...Per Christum Dominum nostrum. Amen.

Por último, en la tablilla derecha aparece el Credo, en 32 líneas de texto, acompañado de notación cuadrada en la primera frase, con dos melodías diferentes para poder adaptarlo a la festividad en la que se cante.

El análisis de la tipografía, siguiendo el método Proctor Haebler de las 20 líneas de texto, ${ }^{45}$ presenta algunas dificul-

45 Esta forma de clasificación de los tipos incunables fue desarrollado por Robert Proctor en 1898 y consiste en organizarlos en función de su tamaño en milímetros, tomando como medida veinte líneas de texto impreso. Konrad Haebler en 1905 añadió el uso de la M mayúscula como letra testigo para diferenciar las diversas letrerías. Sobre su uso y problemática cfr. Rial 2012. tades pues no se conservan más que cuatro líneas de la tipografía mayor y además tampoco se localiza $\mathrm{M}$ mayúscula como letra testigo. Mediante la ponderación de las medidas obtenidas, sin embargo, se constata el empleo de un 268G como tipo mayor y un $128 \mathrm{G}$ como tipo menor. Aunque esta combinación de tipos no aparece registrada por los bibliógrafos dedicados a la primera mitad del siglo XVI, en especial, Frederick J. Norton, el taller zaragozano de Jorge Coci sí emplea una 268G. Además la comparación visual de los tipos empleados, pese al desgaste que muestra la sacra de la Biblioteca Nacional, confirma esta vinculación (Fig. 14).

FIGURA 14

Comparativa de algunos tipos empleados en la sacra de la BNE y los usados por el taller zaragozano de Jorge Coci en otros impresos firmados

BNE, R/41699
$\begin{aligned} & \text { 268G del taller } \\ & \text { de Jorge Coci }\end{aligned}$

El tercero de los ejemplares pre-tridentinos conocidos es, en realidad, un fragmento de pergamino que corresponde con la parte central de una sacra, impresa probablemente por el mismo taller zaragozano de Jorge Coci. Este ejemplar, identificado recientemente mediante la aplicación del método Proctor-Haebler y la comparación visual con la tipografía usada por el citado taller zaragozano en estos años, ${ }^{46}$ se conserva en el Archivo Histórico Nacional como encuadernación de un grimorio manuscrito, prueba inculpatoria contra un clérigo aragonés acusado de nigromante ante el Tribunal de la Inquisición de Toledo ${ }^{47}$ (Fig. 15).

FIGURA 15

Fragmento de sacra impresa en pergamino

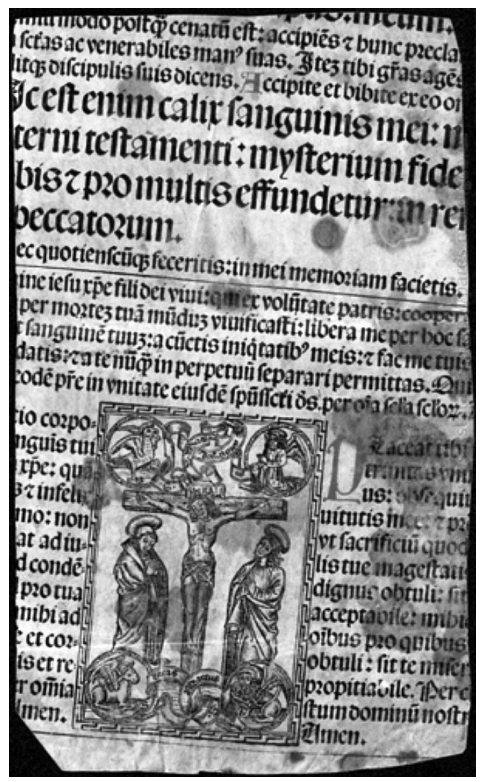

Fuente: Zaragoza, Jorge Coci, AHN, Inquisición, MPD. 442

46 Carvajal 2019.

47 El grimorio, catalogado con el título de Dietario mágico, y el proceso inquisitorial han sido estudiados por Bamford 2018; Morales 2014; Splendiani, Sánchez y Luque 1997, 75. 
Figura 16

Detalle de la iluminación interior y decoración pictórica exterior de la Sacra del Cardenal Rodrigo de Castro, finales S. XVI
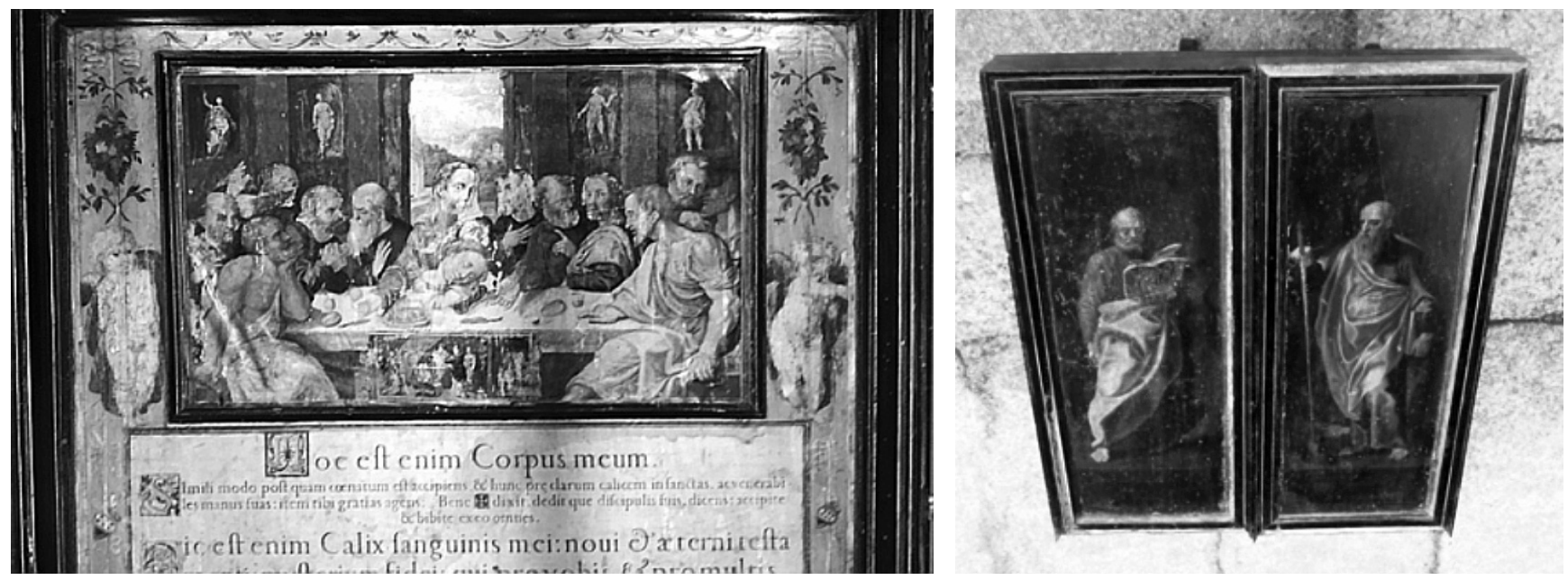

Fuente: Colegio de Nuestra Señora de la Antigua de Monforte de Lemos. Fotografía autora

El análisis de este fragmento permite establecer un tamaño muy similar a la sacra de Villafranca del Cid y algo más pequeño que el de la Biblioteca Nacional de España, aunque con una distribución similar a esta última, similitud que se acentúa por la inclusión del grabado xilográfico. Su interés es doble y radica, por un lado, en que probablemente se corresponda con alguna de las amplias tiradas que de este producto editorial realizaron talleres como el de Jorge Coci y de las que hasta la fecha no se tenía más constancia que la documental antes citada;48 por otro, da buena cuenta de cómo estos objetos fueron reutilizados de las formas más variadas al caer en desuso.

Existe en España otro ejemplar interesante de sacra del siglo XVI, aunque en este caso se realizó probablemente después del Concilio de Trento, indicando la continuidad y vigencia de estos objetos. Se trata de las sacras del Cardenal Rodrigo de Castro (1523-1600), conservadas en el Colegio de Nuestra Señora de la Antigua de Monforte de Lemos, institución educativa de venerable antigüedad, encomendada en su origen a los jesuitas y actualmente gestionada por los Padres Escolapios. ${ }^{49}$ Estas tabellae, de gran tamaño y suntuosidad en comparación con las anteriores, están realizadas sobre pergamino manuscrito adherido a la madera. Lo más llamativo es la rica decoración pictórica tanto del interior, con una escena de la Santa Cena de inspiración italianizante, y los santos Pedro y Pablo que ornamentan el exterior cuando el objeto se halla cerrado (Fig. 16).

\section{A MODO DE CONCLUSIÓN}

Según muestran las fuentes documentales, las sacras fueron un producto de amplia difusión y ubicuidad en los reinos hispanos desde la Baja Edad Media. Su precio modesto, sobre todo después de la aparición y consolidación de la imprenta, facilitó que prácticamente cualquier sede religiosa, por humilde que fuera, pudiera acceder a este producto editorial utilitario.

48 Vid. nota 13.

49 Agradezco al padre Javier Agudo, responsable de patrimonio histórico del Colegio de Nuestra Señora de la Antigua de Monforte de Lemos, su recibimiento y ayuda para el estudio de estas sacras.
El bajo coste de estas piezas, que se pone de manifiesto al establecer la equivalencia de su precio con el de otros objetos cotidianos, explica, además, otros dos hechos llamativos. En primer lugar, justifica el alto grado de desgaste que se aprecia en los materiales tipográficos y xilográficos empleados en la realización de los ejemplares impresos, sobre todo al compararlos con otras producciones contemporáneas del mismo taller. Asimismo, algunos de los grabados empleados son de una calidad artística notablemente inferior a los de igual temática usados por el taller de Coci en otras piezas coetáneas.

Por otra parte, justifica por qué estas piezas, salvo excepciones como la conservada en la BNE y procedente de una colección particular, fueron amortizadas sin remordimientos al caer en desuso, sustituidos por ejemplos más actualizados, a veces reconvertidas en encuadernación y otras sencillamente abandonadas en un desván.

Sin embargo, como ya se ha mencionado, las fuentes documentales indican que la producción de estos objetos fue masiva, igual que sucedió con otros productos editoriales de consumo cotidiano como las estampas y las bulas, afectadas también por un altísimo índice de desaparición. Dada la escasa pervivencia de ejemplos hispanos medievales y modernos tempranos cabe preguntarse si estas hojas con oraciones, presentes tanto en las fuentes documentales como icónicas de la Baja Edad Media y el tránsito a la modernidad, han podido catalogarse erróneamente como folios de manuscritos, perdiéndose de este modo la vinculación con su uso primitivo. No hay que descartar, por tanto que muchos otros ejemplares puedan ir apareciendo reutilizados de diversas formas, como sucede con el citado fragmento del Archivo Histórico Nacional.

\section{BibLIOGRAFÍA}

Abizanda y Broto, Manuel. 1915-1930, Documentos para la historia artística y literaria de Aragón procedentes del archivo de Protocolos de Zaragoza, siglo XVI. Zaragoza: La editorial.

Álvarez Márquez, María del Carmen. 1992a. El mundo del libro en la iglesia catedral de Sevilla en el siglo XVI. Sevilla: Diputación Provincial. 
Álvarez Márquez, María del Carmen. 1992b. «La formación de los fondos bibliográficos de la Catedral de Sevilla». En El libro antiguo español. Actas del segundo Coloquio Internacional, ed. María Luisa López-Vidriero y Pedro Manuel Cátedra. Salamanca: Universidad de Salamanca.

Bamford, Heather. 2018. "Unprinted: Magic, Reading and Meaning in Early Modern Iberian Manuscript Text». eHumanista/Conversos 6: 403-418.

Brancato Domenica y Giuseppe Pontillo. 2016. Fasto in liturgia. Paramenti e suppellettili sacre del vescovo Lucchesi Palli. Agrigento: Ecclesiaviva Edizioni.

Brouns, Benoit. 2005. Paisatges sagrats: la llum de les imatges. San Mateu 2005. Valencia: Generalitat Valenciana, Conselleria de Cultura, Educació i Esport.

Carvajal González, Helena. 2019. "CCient sacras de pargamino": Un impreso "sine notis" desconocido del taller zaragozano de Jorge Coci en el Archivo Histórico Nacional». Revista General de Información y Documentación 29 (2): 413-425.

Castillo Gómez, Antonio. 2006. Entre la pluma y la pared: una historia social de la escritura en los siglos de oro. Madrid: Akal.

Castillo Gómez, Antonio. 2009. "La letra en la pared. Usos y funciones de la escritura expuesta en el Siglo de Oro». En Testigo del tiempo, memoria del universo: cultura escrita y sociedad en el mundo ibérico (siglos XV-XVIII), coord. Manuel Fernández, Carlos-Alberto GonzálezSánchez y Natalia Maillard Álvarez, 581-602. Málaga: Rubeo.

Feliu, Gaspar. 1991. Precios y salarios en la Cataluña moderna. Madrid Banco de España.

Ferrandis, José. 1943. Datos documentales para la historia del arte español. Madrid: Consejo Superior de Investigaciones Científicas, Centro de Estudios Históricos.

García-Moreno y Martínez, Manuel. 1902. «Inventario de la Catedral de Salamanca (año 1275)» Revista de Archivos, Bibliotecas y Museos Año VI, VII: 175-179.

Griffin, Clive. 1988. «Un curioso inventario de libros de 1528». En El libro antiguo español: actas del Primer Coloquio Internacional, ed. Pedro Manuel Cátedra y María Luisa López-Vidriero, 189-224. Salamanca: Universidad de Salamanca.

Giorgi, Rosa. 2008. The History of the Church in Art. Los Ángeles: Getty Publications.

Jungmann Josef Andreas. 1962. Missarum Sollemnia. Eine genetische Erklärung der römischen Messe. Wien - Freiburg - Basel: Herder Verlag.

Lecuona, Manuel de. 1952. "La parroquia de Santiago de Calahorra: breves notas históricas». Berceo 24: 469-490.

Llopis Enrique y Elisa Ruiz. 2019. El monasterio de Guadalupe y la Inquisición. Madrid: Ediciones Complutense.

Martí I Bonet, Josep María. 2013. Sacralia Antiqua. Diccionari del catalogador del patrimoni cultural de l'Església. Barcelona: Arxiu Diocesà de Barcelona.

Martín Nieto, Dionísio. 2014. "Santa Lucía de Alcuéscar, olim San Salvador de los Monesterios». Revista de Estudios Extremeños: LXX, III: 1525-1574.

Milián Boix, Manuel. 2013. Inventario monumental dertusense: Diócesis de Tortosa. [Valencia]: Generalitat Valenciana, Conselleria d'Educació, Cultura i Esport - Castelló de la Plana: Diputació de Castelló.
Monferrer I Guardiola, Rafael. 1986. El temple parroquial de Vilafranca. Castelló de la Plana: Societat Castellonenca de Cultura.

Morales Estévez, Roberto. 2014. "Los grimorios y recetarios mágicos: Del mítico Salomón al clérigo nigromante». En Señales, Portentos y Demonios. La magia en la literatura y la cultura españolas del Renacimiento, coord. Eva Lara y Alberto Montaner. Salamanca: SEMYR.

Oppenheim, Filippo. 1949. "Cartegloria». En Enciclopedia Cattolica, III, coll. 956-957. Città del Vaticano.

Ortiz Rico, Isabel María. 1995. «Libros litúrgicos de iglesias y ermitas en las encomiendas santiaguistas (1507)». SIGNO. Revista de Historia de la Cultura Escrita 2: 55-75.

Pedraza Gracia, Manuel José. 1991. La imprenta de Gabriel de Híjar. Zaragoza: Institución Fernando el Católico.

Pedraza Gracia, Manuel José. 2016. «Por George Coci alemán». En La literatura medieval hispánica en la imprenta (1475-1600), ed. María Jesús Lacarra y Nuria Aranda García, 201-214. Valencia: Universitat de València, Servei de Publicacions.

Pomar Rodil, Pablo Javier. 2012. «El uso litúrgico de los atriles de altar en el culto católico de ámbito hispano». En Estudios de platería, San Eloy 2012, coord. Jesús Rivas Carmona, 475-490. Murcia: Universidad de Murcia, Servicio de Publicaciones.

Rial Costas, Benito. 2012. «El sistema Proctor-Haebler y el estudio de las letrerías en las impresiones góticas incunables». En Literatura medieval y renacentista en España: líneas y pautas, coord. Natalia Fernández Rodríguez y María Fernández Ferreiro, 855-864. Salamanca: SEMYR.

Riesco Terrero, Ángel. 1996. «Un inventario de la catedral de Salamanca del siglo XIII». Espacio, Tiempo y Forma. Serie III, Historia Medieval 9: 277-302.

Righetti, Mario. 1955. Historia de la liturgia. Madrid: La Editorial Católica.

Ruiz García, Elisa. 2004. "Los breviarios de la reina católica: un signo de modernidad». En III Jornadas Científicas Sobre Documentación en época de los Reyes Católicos: 221-248. Madrid: Universidad Complutense de Madrid. En línea https://www.ucm.es/data/cont/ docs/446-2013-08-22-9\%20breviarios.pdf

Schmidt, Peter. 2010. "Liturgische Einblattdrucke Neue Funde und Überlegungen zur Frühgeschichte der Kanontafel im 15.und 16. Jahrhundert». Gutenberg-Jahrbuch: 25-42.

Schulte Agustin Joshep 1913. "Cartagloria». En Catholic Encyclopedia, vol. I. New York: The Encyclopedia Press.

Serrano y Sanz, Manuel. 1915. «Documentos. Inventarios aragoneses de los siglos XIV y XV. Inventarios litúrgicos de algunas iglesias que pertenecían a las órdenes de Santiago y Calatrava». Boletín de la Real Academia Española II: 44-48.

Solórzano Tellechea, Jesús Angel. 2007. Documentación medieval en la Biblioteca Municipal de Santander. Manuscritos originales (945 1519). Santander: Asociación Cántabra de Estudios Medievales.

Splendiani, Anna María, José Enrique Sánchez Bohórquez y Emma Cecilia Luque De Salazar. 1997. Cincuenta años de inquisición en el Tribunal de Cartagena de Indias, 1610-1660. Bogotá: Pontificia Universidad Javeriana.

Villamil y Castro José de. 1906. Inventarios de mobiliario litúrgico. Madrid: Nueva imprenta de San Francisco de Sales. 